Simon R. Mucha, MD Critical Care Medicine, Respiratory Institute, Cleveland Clinic; Clinical Instructor, Cleveland Clinic Lerner College of Medicine of Case Western Reserve University, Cleveland, $\mathrm{OH}$

\section{John Bartholomew, MD} Cardiovascular Medicine, Cleveland Clinic; Professor, Cleveland Clinic Lerner College of Medicine of Case Western Reserve University, Cleveland, $\mathrm{OH}$
Siddharth Dugar, MD Pulmonary Medicine, Cleveland Clinic; Clinical Assistant Professor, Cleveland

Clinic Lerner College of Medicine of Case Western Reserve University,

Cleveland, $\mathrm{OH}$
Keith McCrae, MD Hematology and Medical Oncology, and Cellular and Molecular Medicine, Cleveland Clinic:

Professor, Cleveland Clinic Lerner College of Medicine of Case Western Reserve University Cleveland, $\mathrm{OH}$
Douglas Joseph, DO

Cardiovascular Medicine, Cleveland Clinic; Assistant Professor, Cleveland Clinic Lerner College of Medicine of Case Western Reserve University, Cleveland, $\mathrm{OH}$
Gretchen L. Sacha, PharmD, RPh, BCCCP

Inpatient Pharmacy, Cleveland Clinic
Michael Militello, PharmD, RPh, BCPS Inpatient Pharmacy, Cleveland Clinic

\title{
Update to coagulopathy in COVID-19: Manifestations and management
}

\section{Posted December 12, 2020}

\section{ABSTRACT}

Severe COVID-19 illness is associated with intense inflammation, leading to high rates of thrombotic complications that increase morbidity and mortality. Markedly elevated levels of D-dimer with normal fibrinogen levels are the hallmark laboratory findings of severe COVID-19-associated coagulopathy. Prophylaxis against venous thromboembolism is paramount for all hospitalized patients with COVID-19, with more aggressive prophylaxis and screening recommended for critically ill patients with D-dimer levels above $3.0 \mu \mathrm{g} / \mathrm{mL}$. Point-of-care ultrasonography is the imaging method of choice for patients at high risk, as it entails minimal risk of exposing providers to the virus.

\section{KEY POINTS}

We recommend measuring $\mathrm{D}$-dimer, fibrinogen, prothrombin time, international normalized ratio, and activated partial thromboplastin time every 48 hours in hospitalized patients with COVID-19.

Prophylaxis against venous thromboembolism is recommended for all patients with COVID-19 on admission, using low-molecular-weight heparin, unfractionated heparin for those in renal failure, or fondaparinux for those with heparin-induced thrombocytopenia, even in the setting of thrombocytopenia as long as the platelet count is above $25 \times 10^{9} / \mathrm{L}$.

Critically ill intensive care unit patients with D-dimer levels 3.0 $\mu \mathrm{g} / \mathrm{mL}$ or higher should undergo screening with point-of-care ultrasonography and receive more intensive prophylaxis.

The statements and opinions expressed in COVID-19 Curbside Consults are based on experience and the available literature as of the date posted. While we try to regularly update this content, any offered recommendations cannot be substituted for the clinical judgment of clinicians caring for individual patients.

doi:10.3949/ccjm.87a.ccc024-up

\section{INTRODUCTION}

COVID-19-associated coagulopathy (CAC) and disseminated intravascular coagulation are common in COVID-19 and are associated with severe illness and death. ${ }^{1-3}$ Critically ill patients without other risk factors for thrombosis can experience various thrombotic events, including microvascular thrombosis, venous and pulmonary thromboembolism, and acute arterial thrombosis. ${ }^{4}$

This article discusses clinical manifestations of CAC, associated laboratory and histologic findings, recent evidence elucidating pathophysiologic mechanisms, and the way we manage it at Cleveland Clinic.

\section{A HIGHLY THROMBOTIC STATE}

The clinical presentation of CAC is that of a highly thrombotic state. Shared anecdotal experience from a variety of sources indicates that catheter-associated thrombosis and clotting of vascular access catheters are frequently encountered. The need for catheter replacement and dialysis circuits that involve frequent interruption of continuous renal replacement therapy are other scenarios at high-risk for clotting and thrombosis development.

Two earlier studies support the clinical impression that COVID-19 is highly thrombotic. Cui et al ${ }^{5}$ reported a $25 \%$ incidence of deep vein thrombosis in patients with severe coronavirus pneumonia in Wuhan. In the Netherlands, Klok et $\mathrm{al}^{4}$ found a 31\% combined incidence of deep vein thrombosis, pulmonary embolism, and arterial thrombosis in critically ill patients with coronavirus. Of these events, $81 \%$ were pulmonary thromboemboli. This is similar to our own clinical experience at Cleveland Clinic intensive care units (ICUs), where the prevalence of DVT is $25 \%$ to $30 \%$ on point of care ultrasound examination (POCUS). Although variable prevalence rates have 
been reported in subsequent studies, a recent metaanalysis confirmed a pooled prevalence rate of venous thromboembolism (VTE) of $31 \%$ in ICU patients despite pharmacologic VTE prophylaxis. ${ }^{6}$ Another frequent finding observed on POCUS is occurrence of spontaneous echo contrast (SEC); described as amorphous echogenicity in major veins. The presence of SEC has been associated with a higher subsequent risk of deep vein thrombosis (Figures 1-3). ${ }^{7,8}$

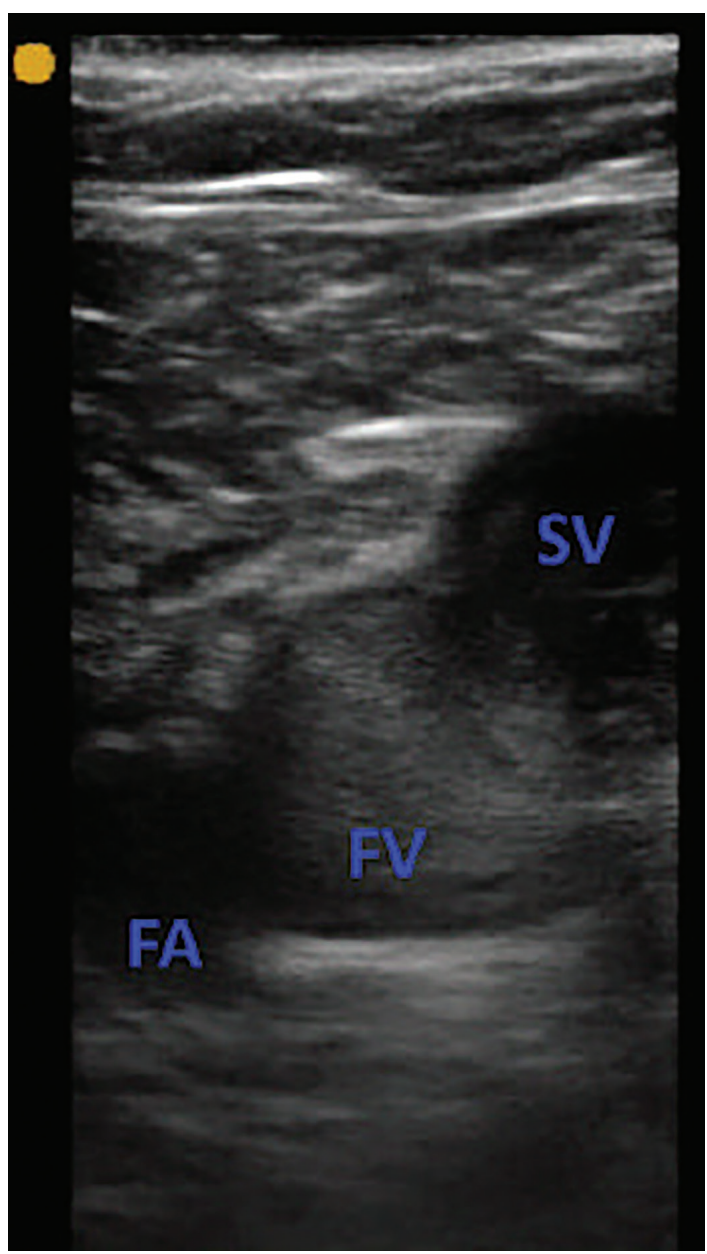

Figure 1. A short-axis view of the femoral vein (FV) and the femoral artery (FA) at the site of the saphenous vein (SV) inflow. Amorphous echogenicity in the femoral vein, greater than that of the adjacent femoral artery, is suggestive of slow venous flow. The vein was fully compressible, ruling out deep vein thrombosis at the site.

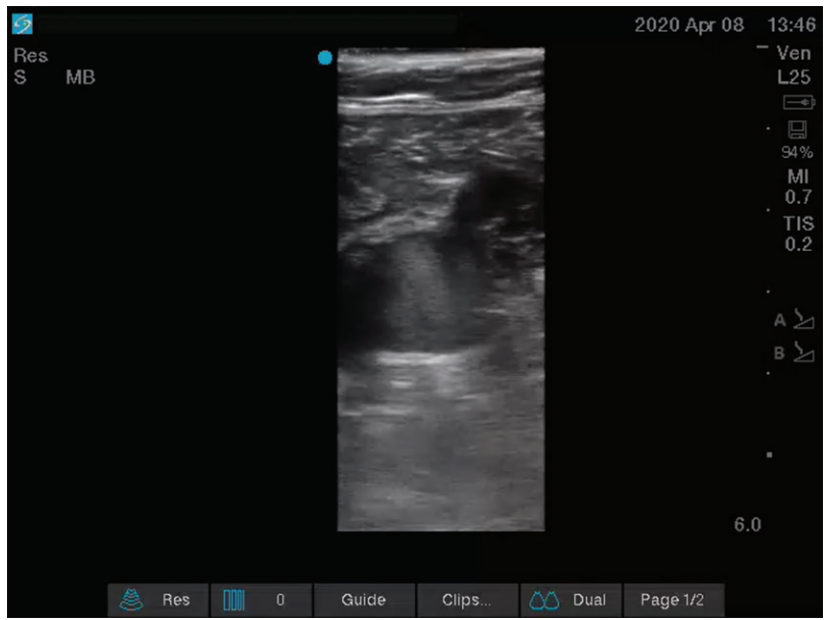

Figure 2. Another short-axis view of the femoral vein (center) and the femoral artery (bottom right) at the site of the saphenous vein inflow (top right). Swirling pattern of high echogenicity suggests lowflow state.

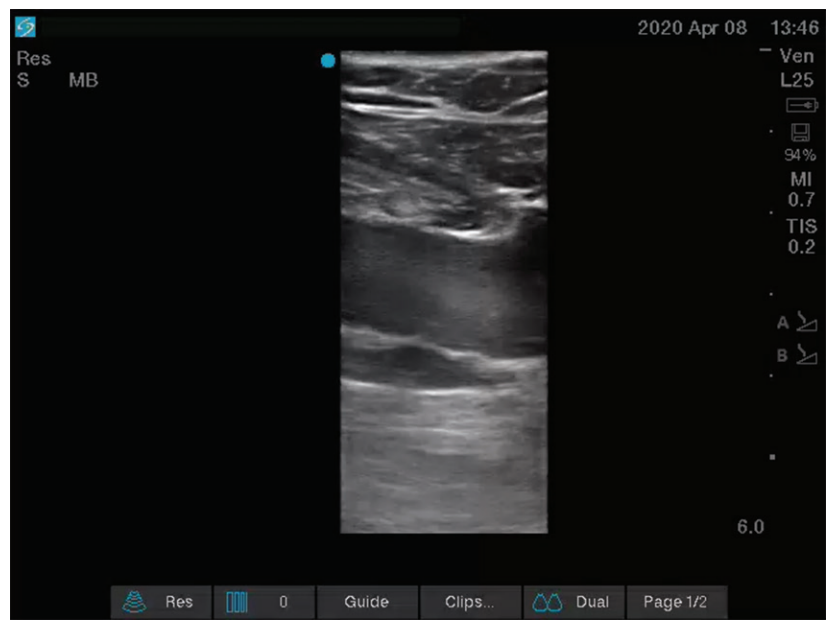

Figure 3. Long-axis view of the femoral vein, with spontaneous echogenicity and slow flow.

\section{LABORATORY FINDINGS: ELEVATED D-DIMER}

The characteristic laboratory findings of CAC are significantly elevated levels of D-dimer, fibrin degradation products and fibrinogen, indicating a highly thrombotic state with high fibrin turnover. ${ }^{9}$ However, other markers of disseminated intravascular coagulation remain relatively unchanged. ${ }^{10}$ The prothrombin time and activated partial thromboplastin time are only mildly prolonged, if at all, and platelet counts are usually normal or marginally reduced (100-150 × $\left.10^{9} / \mathrm{L}\right) .{ }^{9,11,12}$ Abnormalities in coagulation parameters 
have been shown to correlate directly with inflammatory cytokines, severity of illness and adverse outcomes. ${ }^{13,14}$

\section{Elevated D-dimer}

Elevated D-dimer levels on presentation with COVID-19 are a hallmark of more severe disease. Levels of $0.5 \mu \mathrm{g} / \mathrm{mL}$ or higher were found in $59.6 \%$ of patients with severe disease vs $43.2 \%$ of those with mild disease. ${ }^{3}$ High levels also correlated with the need for intensive care ${ }^{15}$ and with death.

In a multivariable regression analysis of 191 patients, Zhou et $\mathrm{al}^{1}$ reported that the risk of death was more than 18 times higher (odds ratio 18.42, 95\% confidence interval 2.64-128.55) for patients admitted with a D-dimer level greater than $1 \mu \mathrm{g} / \mathrm{mL}$ vs less than $0.5 \mu \mathrm{g} / \mathrm{mL}$. Cui et $\mathrm{al}^{5}$ reported that D-dimer levels also correlated with risk of venous thromboembolism: a level of $3.0 \mu \mathrm{g} / \mathrm{mL}$ had a sensitivity of $70.0 \%$, specificity of $96.7 \%$ with a positive predictive value of $87.5 \%$. Maatman et $\mathrm{al}^{16}$ reported that standard prophylaxis against venous thromboembolism failed in 29 of 109 patients in the ICU, and of those in whom it failed, all had D-dimer levels greater than $3.0 \mu \mathrm{g} / \mathrm{mL}$.

\section{Fibrinogen}

Fibrinogen is characteristically elevated in patients with severe COVID-19. Fibrinogen levels correlate with levels of IL-6 ${ }^{17}$ and severity of disease. ${ }^{18}$ Levels appear to be elevated throughout the course of the disease, but an acute drop late in the course may signal the onset of acute consumptive coagulopathy and be an indicator of particularly poor prognosis. ${ }^{9}$

\section{Prothrombin time, activated partial thromboplastin time}

Klok et $\mathrm{al}^{4}$ did not report D-dimer levels, but found coagulopathy (ie, prolongation of prothrombin time of $>3$ seconds or of activated thromboplastin time $>5$ seconds) to be an independent risk factor for thrombosis. Prolongation of PT has also been shown to correlate with more severe disease, greater risk for organ failure (such as ARDS and renal failure) and increased mortality. ${ }^{13}$

\section{Antiphospholipid antibodies}

Zhang et $\mathrm{al}^{19}$ reported that 3 patients with CAC and lower-extremity ischemia had antiphospholipid antibodies (anticardiolipin immunoglobulin A [IgA], anti-beta-2 glycoprotein $1 \mathrm{IgA}$ and $\mathrm{IgG}$ ) but not lupus anticoagulant. Helms et $\mathrm{al}^{20}$ in a multicenter study of 150 patients with COVID-19 in ICUs in
France, found a remarkably high rate of positivity for lupus anticoagulant: 50 of 57 patients $(87.7 \%)$ among those tested for further evaluation for an elevated activated partial thromboplastin time. Preliminary studies suggest that these antibodies may induce neutrophil extracellular trap release from neutrophils. ${ }^{21}$

\section{Disseminated intravascular coagulation score}

Tang et $\mathrm{al}^{9}$ found that progression of coagulopathy to overt disseminated intravascular coagulation (defined by the International Society on Thrombosis and Haemostasis as a disseminated intravascular coagulation score $\geq 5$ points; the score is based on platelet count, D-dimer level, fibrinogen level, and prolongation of the prothrombin time) predicted a poor prognosis, occurring in $71.4 \%$ of all nonsurvivors vs $0.6 \%$ of survivors.

\section{Progressive consumptive coagulopathy}

Declining levels of antithrombin III, a rise in prothrombin time and activated partial thrombolastin time, and dramatic further increase of D-dimer (> $15.0 \mu \mathrm{g} / \mathrm{mL}$ ) appear to indicate severe and progressive disease, developing late in the disease course (day 10 to 14) of nonsurvivors. Fibrinogen levels, which are elevated in the initial phase, drop late in the course of disease in nonsurvivors and may signal impending death. ${ }^{9}$

\section{Low platelet count}

Lippi et al, ${ }^{11}$ in a meta-analysis of 9 studies with 1,779 patients with COVID-19, examined thrombocytopenia as a marker of disease severity. Thrombocytopenia at presentation was associated with an increased risk of severe disease and death, with a weighted mean difference of $31 \times 10^{9} / \mathrm{L}$ in the platelet count between those with severe and nonsevere disease. The authors noted great heterogeneity among studies, with reported rates of thrombocytopenia in severe disease ranging from $4 \%$ to $57.7 \%$.

\section{Viscoelastic testing}

Viscoelastic test such as Rotational Thromboelastometry (ROTEM) and Thromboelastography (TEG) are "global" tests of clot formation, clot strength and clot lysis. Several studies have shown a "hypercoagulable profile" on viscoelasctic testing, including faster clot formation, increased clot strength and a decrease in clot lysis. ${ }^{17,22-25}$ A hypercoagulable profile on viscoelastic testing may be associated with an increased risk of thrombosis despite adequate prophylaxis and organ failure. 


\section{SEVERE LUNG DAMAGE FROM INFLAMMATION, THROMBOSIS}

Histopathologic studies reveal diffuse alveolar damage with profound inflammation, thrombosis, and thrombotic microangiopathy of small vessels and capillaries of the lung. Also noted have been megakaryocytes within pulmonary capillaries with nuclear hyperchromasia and atypia, as well as neutrophils partially degenerated and entrapped in fibers (suggesting neutrophil extracellular traps). ${ }^{26}$ An autopsy series of 11 patients showed thrombosis of small and midsized pulmonary arteries in all patients. ${ }^{27}$

Endothelial cell injury and diffuse microvascular thrombosis suggestive of thrombotic microangiopathy have also been reported in extrapulmonary organs, which may explain the acute onset of multiorgan failure without an otherwise obvious etiology. ${ }^{28}$

\section{PATHOPHYSIOLOGY: INFLAMMATION PROMOTES THROMBOSIS}

CAC is likely multifactorial, and patients with COVID-19 share many of the classic risk factors for venous thromboembolism seen in adult respiratory distress syndrome from other causes, such as immobility, large vascular-access catheters, and systemic inflammation.

The hallmark of COVID-19 is profound inflammation, described as "cytokine storm," characterized by high levels of interleukin 1 (IL-1), IL-6, tumor necrosis factor, and other inflammatory cytokines. ${ }^{15}$ Inflammation promotes thrombosis through various mechanisms, including activation of endothelial cells, platelets, monocytes, and the tissue factorfactor VIIa pathway, and by altering fibrinolysis and natural anticoagulant pathways (eg, through changes in levels of thrombomodulin, proteins $\mathrm{C}$ and $\mathrm{S}$, and tissue-factor-pathway inhibitor). ${ }^{29,30}$

Multiple studies have demonstrated a direct correlation between cytokine levels and laboratory marketers of coagulopathy coagulopathy. ${ }^{13,14}$ Intense inflammation with thrombosis of pulmonary vessels is also seen in adult respiratory distress syndrome of other etiologies. ${ }^{31}$ It remains to be seen if these findings represent a distinct phenotype unique to COVID-19 or are a general indicator of the severity of inflammation with COVID-19.

Serum proteomic profiling of patients with severe acute respiratory syndrome (SARS) identified an $\mathrm{N}$-terminal fragment of complement C3C-alpha (a central component of the complement pathway) as a sensitive biomarker of early SARS. ${ }^{32}$ Murine mod- els of SARS and Middle East respiratory syndrome (MERS) have shown that complement activation is a major contributor to lung injury and other organ failure. Complement inhibition in these models reduced organ damage and inflammation. ${ }^{33,34}$ Complement inhibition has been suggested as a treatment for COVID-19, but clinical data are not yet available..$^{35}$ The SARS-CoV-2 spike protein activates complement in vitro via the alternative pathway. ${ }^{36}$

One mechanism of microvascular thrombosis that may be specific to COVID-19 is the virus's affinity for angiotensin-converting enzyme 2, which is expressed on alveolar epithelial type II cells and various extrapulmonary tissues, including endothelial cells. SARS-CoV-2 has been shown to directly invade the endothelial cell. ${ }^{37}$ Endothelial cell activation may be a unique mechanism of COVID-19-mediated microvascular injury, thrombosis, and subsequent multisystem organ failure and is believed to be a major contributor to coagulopathy, morbidity and mortality. ${ }^{37-40}$

The rate of $87.7 \%$ positivity for lupus anticoagulant in patients with COVID-19 reported by Helms et $\mathrm{a}^{20}$ is striking and needs to be verified, but it supports the idea that endothelial injury is a key mechanism of multiorgan failure and coagulopathy in this disease. The "two-hit" model of thrombosis associated with antiphospholipid syndrome proposes that after a first-hit injury to the endothelium, antiphospholipid antibodies potentiate thrombus formation as a second hit. ${ }^{41}$ Activation of the contact system due to increased vascular permeability and thrombotic microangiopathy warrant further exploration. ${ }^{42}$

\section{OUR MANAGEMENT APPROACH}

Currently, CAC is managed largely on the basis of anecdotal experience and cohort studies; controlled studies are currently underway but high quality prospective data is still lacking and is urgently needed to better guide care.

This lack of data is reflected in the conflicting recommendations from the various guidelines for the management of thrombotic risk associated with severe COVID-19.

The current CHEST guidelines recommend standard VTE prophylaxis. ${ }^{43}$ In contrast, an expert panel of the American College of Cardiology failed to reach consensus on a recommendation for standard versus intensified prophylaxis or empiric therapeutic dose anticoagulation, ${ }^{44}$ while the authors of the interim guidance from the anticoagulation forum recommend high intensity prophylactic dosing for all critically ill patients. ${ }^{45}$ Our approach represents an attempt to 
balance the potential risks and benefits of intensified prophylaxis by selecting patients at greatest risk for thrombosis for intensified prophylaxis. As we await the results of prospective randomized controlled trials, we are assured by recent reports of low overall bleeding risk even with empiric therapeutic anticoagulation. ${ }^{46}$

\section{Monitor D-dimer, fibrinogen, prothrombin time, activated partial thromboplastin time}

In view of the characteristic laboratory findings of CAC described above, we monitor D-dimer, fibrinogen, prothrombin time-international normalized ratio, and activated partial thromboplastin time every 48 hours. We define a D-dimer level of at least 6 times the upper limit of normal $(3.0 \mu \mathrm{g} / \mathrm{mL}$ fibrinogen equivalent units [FEU]) as high risk. ${ }^{5,9}$

Because antiphospholipid antibodies, including lupus anticoagulant, have been reported in COVID19 , we recommend testing for these if the activated partial thromboplastin time is spontaneously elevated, and we prefer the use of anti-Xa assays to monitor anticoagulation in patients on therapeutic anticoagulation. Anti-Xa assays however, may be affected by high levels of bilirubin $(>6.6 \mathrm{mg} / \mathrm{dL})$ or triglycerides $(>360 \mathrm{mg} / \mathrm{dL}),{ }^{47}$ which are often elevated in patients with COVID-19 and cytokine storm. Adding to the risk of hypertriglyceridemia is the use of propofol, a lipid emulsion, for sedation in mechanically ventilated patients. Triglyceride levels should therefore be monitored routinely and considered as a possible source of error in patients on anticoagulation who are difficult to maintain within the therapeutic target range.

A hypercoagulable pattern on viscoelastic testing (thromboelastography or rotational thromboelastometry), with faster time to clot formation, rapid clot propagation, and increased clot strength, has been described in several publications. ${ }^{16,17}$ However, no evidence exists on how to best use this information to guide therapy. In line with current recommendations from various societies and expert panels, we do not routinely use viscoelastic testing to assess hypercoagulability. ${ }^{43,48}$

\section{Imaging: Use POCUS}

To limit caregiver exposure, we minimize formal bedside vascular studies and sending the patient out of the ICU for computed tomographic angiography. We rely heavily on POCUS to assess for evidence of venous thromboembolism. ${ }^{49}$ This is in line with recent recommendations by CHEST and the National Institutes of Health guidance, ${ }^{43,50}$ which cites a lack of evidence supporting routine screening examinations but highlights the value of POCUS in the hands of experienced clinicians. POCUS should be bundled with other care (for example, ultrasonography-guided vascular access) to minimize the use of personal protective equipment and caregiver exposure to COVID-19.

We perform POCUS evaluation in patients with new or acutely worsening hypoxia as well as those who fail to improve or have laboratory indicators of poor prognosis or increase risk of thrombosis. We have defined an elevated D-Dimer level $>6 x$ the upper limit of normal (D-dimer $>3.0 \mu \mathrm{g} / \mathrm{mL}$ FEU) as high risk.

Patients at high risk are assessed for deep vein thrombosis using a 3-point compression POCUS examination of both lower extremities. A POCUS deep vein thrombosis examination and echocardiography are also recommended for any patient with sudden cardiopulmonary decline that cannot be explained by an alternative etiology.

A positive POCUS examination for deep vein thrombosis is highly specific and does not need to be confirmed by formal vascular ultrasonography. ${ }^{51} \mathrm{On}$ the other hand, given the high incidence of pulmonary embolism described, confirmatory studies (ie, formal vascular ultrasonography or computed tomographic angiography) are warranted if the patient has contraindications to empiric anticoagulation and the clinical suspicion of venous thromboembolism is high despite negative POCUS, or if POCUS is not available.

\section{Prophylactic heparin for most}

Specific data on the management of CAC are extremely limited, but given the high risk of thrombotic complications, heparin or low-molecular-weight heparin prophylaxis is currently recommended for all acutely hospitalized patients who do not have specific contraindications.

In addition to its antithrombotic effect, heparin may have anti-inflammatory, anticomplement, ${ }^{52}$ and direct antiviral effects that may be beneficial in COVID-19. Heparin inhibits neutrophil activation, binds inflammatory cytokines, and reduces endothelial activation. ${ }^{53}$ Experimental models have also shown that heparin directly binds to SARS-CoV spike protein, the viral anchor site, thereby blocking viral entry into the cell. ${ }^{54}$ While promising, these effects have yet to be demonstrated clinically.

Tang et $\mathrm{al}^{55}$ reported on 449 patients with severe COVID-19 in whom the overall mortality rate was no 
different $(29.7 \%$ vs $30.3 \%, P=.910)$ between those who received heparin (94 patients on low-molecularweight heparin, 5 patients on unfractionated heparin; prophylactic doses) and those who did not. But among patients with a D-dimer level of more than 6 times the upper limit of normal $(>3.0 \mu \mathrm{g} / \mathrm{mL})$, heparin recipients had a significantly lower mortality rate than nonrecipients $(32.8 \%$ vs $52.4 \%, P=.017)$. The authors concluded that heparin lowers mortality rates in patients with severe COVID-19 and cited a Chinese consensus statement recommending anticoagulation in severe COVID-19. We emphasize that this study retrospectively compared heparin prophylaxis with no prophylaxis.

\section{Full anticoagulation for some?}

Some evidence indicates a higher rate of "heparin failure" in patients with COVID-19. ${ }^{56}$ Elevated D-dimer levels may predict higher risk of venous thromboembolism despite standard prophylaxis. In a study of 240 critically ill patients with COVID-19, Maatman et al ${ }^{16}$ reported a $28 \%$ rate of venous thromboembolism in patients receiving standard prophylaxis. Elevated D-dimer (> 2.6 $\mathrm{\mu g} / \mathrm{mL}$ ) predicted venous thromboembolism with a sensitivity of $89.7 \%$. The authors concluded that standard prophylactic anticoagulant doses may be insufficient to prevent venous thromboembolism in high-risk patients.

Paranjpe et $\mathrm{al}^{57}$ in an observational report of 2,773 patients with COVID-19 admitted to a single institution in New York, found that those treated with full anticoagulation (786 patients, 28\%) had a similar mortality rate $(22.5 \%)$ vs those treated with prophylaxis only $(22.8 \%)$. But among mechanically ventilated patients, in-hospital mortality was $29.1 \%$ for those treated with anticoagulation vs $62.7 \%$ for patients who did not receive anticoagulation. Despite this dramatic reduction of mortality, the authors advise caution in applying these findings, given the serious limitations of the report, ie, its observational nature and lack of information on illness severity and indications for anticoagulation.

Taken together, this limited evidence confirms that prophylaxis against venous thromboembolism in critically ill COVID-19 patients is associated with improved outcomes, and there may be a role for intermediate dose or even full anticoagulation.

Given the limitations of the studies thus far, it remains unclear if higher prophylactic doses, intermediate doses or full anticoagulation, offer a benefit beyond standard prophylactic dosing, and which patients may benefit without suffering more bleeding complications.
Thrombolysis has also been suggested for patients whose condition deteriorate despite anticoagulation. Several case reports and case series describe improvement in oxygenation after treatment with thrombolysis in patients with persistent severe hypoxia, markedly elevated $\mathrm{D}$-dimer or proven thromboembolism. ${ }^{58}$ Most reports do not describe significant bleeding complications, but mortality among these critically ill patients remains high. ${ }^{59}$ Clinical trials to evaluate the role of thrombolytic therapy are currently ongoing.

\section{Recommendations}

Given this lack of evidence, the National Institutes of Health, American Society of Hematology, and International Society on Thrombosis and Haemostasis currently do not recommend treatment beyond standard prophylaxis except for an established indication. Prophylaxis against deep vein thrombosis is strongly recommended in all patients on admission, using lowmolecular-weight heparin (or unfractionated heparin in those with renal failure, or fondaparinux in those with heparin-induced thrombocytopenia). Current guidance also stresses that prophylaxis should be continued even in the setting of thrombocytopenia so long as the platelet count is higher than $25 \times 10^{9} / \mathrm{L} .48,60$ Intensified pharmacologic prophylaxis for ICU patients at greatest risk is also recommended by some. ${ }^{45}$

Our current approach is to provide standard dose pharmacological VTE prophylaxis to all patients with COVID-19 that require inpatient admission. In critically ill patients requiring ICU admission, we adjust therapy based on POCUS screening for venous thromboembolism and intensified prophylaxis in high-risk patients (Table 1, Figure 4). We divide patients into 3 categories:

- Category 1: D-dimer less than $3.0 \mu \mathrm{g} / \mathrm{mL}$ FEU and no evidence of venous thromboembolism. Patients receive standard prophylaxis and are monitored using serial $\mathrm{D}$-dimer testing.

- Category 2: D-dimer $3.0 \mu \mathrm{g} / \mathrm{mL}$ FEU or higher, POCUS-negative. Patients receive intensified deep vein thrombosis prophylaxis.

- Category 3: Confirmed thrombosis. Patients receive full anticoagulation.

If the clinical suspicion of venous thromboembolism is high and the patient has no contraindication to anticoagulation, full anticoagulation should be initiated empirically if POCUS or confirmatory tests are not immediately available.

\section{Continuous renal replacement therapy}

Given the high rate of clotting in dialysis circuits, all patients on continuous renal replacement therapy 


\section{TABLE 1}

\section{Cleveland Clinic approach to anticoagulation prophylaxis and management in COVID-19}

\begin{tabular}{|c|c|}
\hline & $\begin{array}{l}\text { Category } 1 \\
\text { D-dimer }<3.0 \mu \mathrm{g} / \mathrm{mL} \text { FEU } \\
\text { Standard prophylaxis }\end{array}$ \\
\hline Standard & $\begin{array}{l}\text { Enoxaparin } 40 \mathrm{mg} \\
\text { subcutaneously every } 24 \text { hours }\end{array}$ \\
\hline \multirow[t]{4}{*}{ Renal failure } & $\begin{array}{l}\mathrm{CrCl} \mathbf{1 0 - 3 0 ~} \mathbf{~ m L / m i n :} \\
\text { Enoxaparin } 30 \mathrm{mg} \\
\text { subcutaneously every } 24 \text { hours }\end{array}$ \\
\hline & $\begin{array}{l}\mathbf{C r C l}<\mathbf{1 0} \mathbf{~ m L} / \mathbf{m i n} \text { or } \mathbf{A K I} \text { : } \\
\text { Unfractionated heparin } 5,000 \mathrm{U} \\
\text { subcutaneously every } 12 \text { hours }\end{array}$ \\
\hline & $\begin{array}{l}\text { CRRT: } \\
\text { Unfractionated heparin } \\
500 \text { U/hour through circuit }\end{array}$ \\
\hline & $\begin{array}{l}\text { Circuit clotting: } \\
\text { IV heparin per ACS nomogram }\end{array}$ \\
\hline
\end{tabular}

\section{Obesity}

Standard

\author{
$>100$ kg: \\ Enoxaparin $40 \mathrm{mg}$ \\ subcutaneously every 12 hours \\ $>120$ kg: \\ Enoxaparin $60 \mathrm{mg}$ \\ subcutaneously every 12 hours
}

$\begin{array}{ll}\text { Renal failure } & \leq \mathbf{1 2 0} \mathbf{~ k g : ~ 7 , 5 0 0 ~ U ~ e v e r y ~} 12 \text { hours } \\ \mathrm{CrCl}<30 \mathrm{~mL} / & >\mathbf{1 2 0} \mathbf{~ k g : ~} 10,000 \mathrm{U} \text { every } 12 \\ \min \text { or } \mathrm{AKI}{ }^{\mathrm{b}} & \text { hours } \\ & \text { CRRT: } \\ & \mathbf{5 0 0 ~ U / h} \text { through circuit }\end{array}$

Circuit clotting:

IV heparin per ACS nomogram ${ }^{\text {a }}$

\section{Category 2}

D-dimer $\geq 3.0 \mu \mathrm{g} / \mathrm{mL}$ FEU

High-intensity prophylaxis

Enoxaparin $40 \mathrm{mg}$

subcutaneously every 12 hours

$\mathrm{CrCl}<\mathbf{3 0} \mathrm{mL} / \mathrm{min}$ or AKI:

Enoxaparin $40 \mathrm{mg}$

subcutaneously every 24 hours

$\mathrm{CrCl}<10 \mathrm{~mL} / \mathrm{min}$ or $\mathrm{AKI}^{\mathrm{a}}$ :

Unfractionated heparin 7,500 U

subcutaneously every 12 hours

\section{CRRT:}

Unfractionated heparin

$500 \mathrm{U} /$ hour through circuit

\section{Circuit clotting:}

IV heparin per ACS nomogram ${ }^{a}$

\section{$>100$ kg:}

Enoxaparin $60 \mathrm{mg}$

subcutaneously every 12 hours

\section{$>120$ kg:}

Enoxaparin $80 \mathrm{mg}$

subcutaneously every 12 hours

$\leq 120$ kg: 7,500 U every 8 hours

> 120 kg: 10,000 U every 8

hours

CRRT:

$500 \mathrm{U} / \mathrm{h}$ through circuit

\section{Circuit clotting:}

IV heparin per ACS nomogram ${ }^{a}$
Category 3

Confirmed VTE

Full anticoagulation

IV heparin per DVT/PE nomogram or enoxaparin $1 \mathrm{mg} / \mathrm{kg}$ subcutaneously every 12 hours

IV heparin per DVT/VTE nomogram

IV heparin per DVT/PE nomogram

Above $150 \mathrm{~kg}$

use unfractionated heparin

IV heparin per DVT/PE nomogram

\author{
or \\ Enoxaparin $1 \mathrm{mg} / \mathrm{kg}$ \\ subcutaneously every 12 hours, \\ up to $150 \mathrm{mg}$
}

aV heparin ACS nomogram: initial dose 60-U/kg bolus, then 12 U/kg/hour; target aPTT 49-67 seconds; target heparin anti-Xa 0.2-0.5 units/mL.

${ }^{\mathrm{D} A K I}$ definition: doubling of creatinine in 48 hours or anuria.

$\mathrm{ACS}=$ acute coronary syndrome; $\mathrm{AKI}=$ acute kidney injury; $\mathrm{aPTT}=$ activated partial thromboplastin time; $\mathrm{CrCl}=$ creatinine clearance; $\mathrm{CRRT}=$ continuous renal replacement therapy; DVT = deep vein thrombosis; $\mathrm{FEU}=$ fibrinogen equivalent units; $\mathrm{IV}$ = intravenous; $\mathrm{PE}$ = pulmonary embolism; $\mathrm{VTE}=$ venous thromboembolism receive unfractionated heparin at a rate of $500 \mathrm{U}$ per hour. If ongoing clotting is observed, we increase systemic heparin to bring the activated partial thromboplastin time into the target range according to an acute coronary syndrome nomogram. The target acti- vated partial thromboplastin time is 49 to 67 seconds, and the goal anti-factor $\mathrm{Xa}$ level is 0.2 to $0.5 \mathrm{IU} / \mathrm{mL}$, but these may be adjusted if clotting continues despite systemic heparin. 


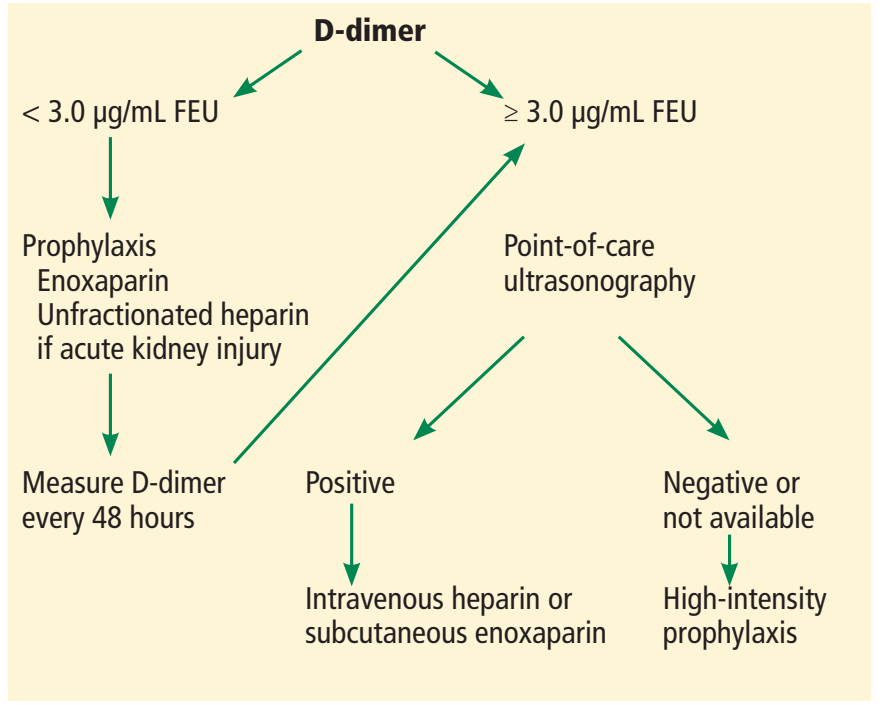

Figure 4. Algorithm for preventing and treating COVID-19-associated coagulopathy.

$\mathrm{FEU}=$ fibrinogen equivalent units

\section{Duration of anticoagulation}

Anticoagulation should be continued for 6 weeks for catheter-associated thrombosis and for at least 3 months for venous thromboembolism. Convalescent patients with persistently elevated D-dimer (greater than twice the upper limit of normal) may benefit from extended prophylaxis or treatment. ${ }^{61,62}$

\section{DISCLOSURES}

The authors report no relevant financial relationships which, in the context of their contributions, could be perceived as a potential conflict of interest.

\section{REFERENCES}

1. Zhou F, Yu T, Du R, et al. Clinical course and risk factors for mortality of adult inpatients with COVID-19 in Wuhan, China: a retrospective cohort study. Lancet 2020; 395(10229): 1054-1062. doi: 10.1016/S0140-6736(20)30566-3

2. Wu C, Chen X, Cai Y, et al. Risk factors associated with acute respiratory distress syndrome and death in patients with coronavirus disease 2019 pneumonia in Wuhan, China. JAMA Intern Med 2020; Mar 13;e200994. doi: 10.1001/jamainternmed.2020.0994

3. Guan WJ, Ni ZY, Hu Y, et al; China Medical Treatment Expert Group for Covid-19. Clinical characteristics of coronavirus disease 2019 in China. N Engl J Med 2020; 382(18): 1708-1720. doi: 10.1056/NEJMoa2002032

4. Klok FA, Kruip MJ, van der Meer NJ, et al. Incidence of thrombotic complications in critically ill ICU patients with COVID-19. Thromb Res 2020; 191:145-147. doi: 10.1016/j.thromres.2020.04.013

5. Cui S, Chen S, Li X, Liu S, Wang F. Prevalence of venous thromboembolism in patients with severe novel coronavirus pneumonia. J Thromb Haemost 2020; 18(6):1421-1424. doi: 10.1111/jth. 14830

6. Hasan SS, Radford S, Kow CS, Zaidi STR. Venous thromboembolism in critically ill COVID-19 patients receiving prophylactic or therapeutic anticoagulation: a systematic review and meta-analysis. J Thromb Thrombolysis. 2020;50(4):814-821. doi:10.1007/s11239-02002235-z
7. Dugar S, Duggal A, Bassel A, Soliman M, Moghekar A. Spontaneous echo contrast in venous ultrasound of severe COVID-19 patients. Intensive Care Med. 2020;46(8):1637-1639. doi:10.1007/ s00134-020-06094-3

8. Jensen CT, Chahin A, Amin VD, et al. Qualitative slow blood flow in lower extremity deep veins on Doppler sonography: quantitative assessment and preliminary evaluation of correlation with subsequent deep venous thrombosis development in a tertiary care oncology center. J Ultrasound Med 2017; 36(9): 1867-1874. doi: 10.1002/jum. 14220

9. Tang N, Li D, Wang X, Sun Z. Abnormal coagulation parameters are associated with poor prognosis in patients with novel coronavirus pneumonia. J Thromb Haemost. 2020;18(4):844-847. doi:10.1111/ jth.14768

10. Yin S, Huang M, Li D, Tang N. Difference of coagulation features between severe pneumonia induced by SARS-CoV2 and non-SARSCoV2. J Thromb Thrombolysis 2020; Apr 3:1-4. doi: 10.1007/s11239020-02105-8

11. Lippi G, Plebani M, Henry BM. Thrombocytopenia is associated with severe coronavirus disease 2019 (COVID-19) infections: a meta-analysis. Clin Chim Acta 2020; 506:145-148. doi: 10.1016/j. cca.2020.03.022

12. Han H, Yang L, Liu R, et al. Prominent changes in blood coagulation of patients with SARS-CoV-2 infection. Clin Chem Lab Med 2020; 58(7): 1116-1120. doi: 10.1515/cclm-2020-0188

13. Liu Y, Gao W, Guo W, et al. Prominent coagulation disorder is closely related to inflammatory response and could be as a prognostic indicator for ICU patients with COVID-19. J Thromb Thrombolysis. 2020;50(4):825-832. doi:10.1007/s11239-020-02174-9

14. Rauch A, Labreuche J, Lassalle F, et al. Coagulation biomarkers are independent predictors of increased oxygen requirements in COVID-19 [published online ahead of print, 2020 Aug 17]. J Thromb Haemost. 2020;10.1111/jth.15067. doi:10.1111/jth.15067

15. Huang C, Wang Y, Li X, et al. Clinical features of patients infected with 2019 novel coronavirus in Wuhan, China. Lancet 2020; 395(10223): 497-506. doi: 10.1016/S0140-6736(20)30183-5

16. Maatman TK, Jalali F, Feizpour C, et al. Routine venous thromboembolism prophylaxis may be inadequate in the hypercoagulable state of severe coronavirus disease 2019. Crit Care Med 2020 May 27; 10.1097/CCM.0000000000004466. doi: 10.1097/ CCM.0000000000004466

17. Ranucci M, Ballotta A, Di Dedda U, et al. The procoagulant pattern of patients with COVID-19 acute respiratory distress syndrome. J Thromb Haemost. 2020;18(7):1747-1751. doi:10.1111/jth.14854

18. Zhang A, Leng Y, Zhang Y, et al. Meta-analysis of coagulation parameters associated with disease severity and poor prognosis of COVID-19 [published online ahead of print, 2020 Sep 15]. Int J Infect Dis. 2020;100:441-448. doi:10.1016/j.ijid.2020.09.021

19. Zhang $\mathbf{Y}$, Xiao M, Zhang S, et al. Coagulopathy and antiphospholipid antibodies in patients with COVID-19. N Engl J Med 2020; 382(17):e38. doi: 10.1056/NEJMc2007575

20. Helms J, Tacquard D, Severac F, et al. High risk of thrombosis in patients in severe SARS-CoV-2 infection: a multicenter prospective cohort study. Intensive Care Med 2020; 46(6): 1089-1098. doi: 10.1007/s00134-020-06062-X

21. Zuo Y, Estes SK, Gandhi AA, Yalavarthi S, Ali RA, Shi H, et al. Prothrombotic antiphospholipid antibodies in COVID-19. medRxiv (2020) 17. doi: 10.1101/2020.06.15.20131607

22. Mortus JR, Manek SE, Brubaker LS, et al. Thromboelastographic Results and Hypercoagulability Syndrome in Patients With Coronavirus Disease 2019 Who Are Critically III. JAMA Netw Open. 2020;3(6):e2011192. Published 2020 Jun 1. doi:10.1001/jamanetworkopen.2020.11192

23. Pavoni V, Gianesello L, Pazzi M, Stera C, Meconi T, Frigieri FC. Evaluation of coagulation function by rotation thromboelastometry in critically ill patients with severe COVID-19 pneumonia. J Thromb Thrombolysis. 2020;50(2):281-286. doi:10.1007/s11239-020-02130-7

24. Wright FL, Vogler TO, Moore EE, et al. Fibrinolysis shutdown correlation with thromboembolic events in severe COVID-19 infection. 
J Am Coll Surg. 2020;231(2):193-203.e1. doi:10.1016/j.jamcollsurg.2020.05.007

25. Stattin K, Lipcsey M, Andersson $\mathrm{H}$, et al. Inadequate prophylactic effect of low-molecular weight heparin in critically ill COVID-19 patients [published online ahead of print, 2020 Sep 3]. J Crit Care. 2020;60:249-252. doi:10.1016/j.jcrc.2020.08.026

26. Fox SE, Akmatbekov A, Harbert JL, Li G, Brown JQ, Vander Heide RS. Pulmonary and cardiac pathology in COVID-19: the first autopsy series from New Orleans. MedRxiv 2020; April 10. doi: 10.1101/2020.04.06.20050575

27. Lax SF, Skok K, Zechner P, et al. Pulmonary arterial thrombosis in COVID-19 with fatal outcome: results from a prospective, singlecenter, clinicopathologic case series. Ann Intern Med 2020 May 14:M20-2566. doi: 10.7326/M20-2566

28. Zhang T, Sun LX, Feng RE. Comparison of clinical and pathological features between severe acute respiratory syndrome and coronavirus disease 2019. Zhonghua Jie He He Hu Xi Za Zhi 2020; 43(6):496502. Chinese. doi:10.3760/cma.j.cn112147-20200311-00312

29. Levi M, Scully M. How I treat disseminated intravascular coagulation. Blood 2018; 131(8):845-854. doi: 10.1182/blood-2017-10804096Abstract/FREE Full Text

30. Engelmann B, Massberg $\mathbf{S}$. Thrombosis as an intravascular effector of innate immunity. Nat Rev Immunol 2013; 13(1):34-45. doi: 10.1038/nri3345

31. Glas GJ, Van Der Sluijs KF, Schultz MJ, Hofstra JJ, Van Der Poll T, Levi M. Bronchoalveolar hemostasis in lung injury and acute respiratory distress syndrome. J Thromb Haemost 2013; 11(1):17-25. doi: 10.1111/jth. 12047

32. Pang RT, Poon CT, Chan KC, et al. Serum proteomic fingerprints of adult patients with severe acute respiratory syndrome. Clin Chem 2006; 52(3): 421-429. doi: 10.1373/clinchem.2005.061689Abstract/ FREE Full Text

33. Gralinski LE, Sheahan TP, Morrison TE, et al. Complement activation contributes to severe acute respiratory syndrome coronavirus pathogenesis. mBio 2018; 9(5). doi:10.1128/mBio.01753-18

34. Jiang Y, Zhao G, Song N, et al. Blockade of the C5a-C5aR axis alleviates lung damage in hDPP4-transgenic mice infected with MERSCoV. Emerg Microbes Infect 2018; 7(1): 77. doi:10.1038/s41426-0180063-8

35. Campbell CM, Kahwash R. Will complement inhibition be the new target in treating COVID-19 related systemic thrombosis? Circulation 2020; 141(22):1739-1741. doi: 10.1161/CIRCULATIONAHA.120.047419

36. Yu J, Yuan X, Chen H, Chaturvedi S, Braunstein EM, Brodsky RA. Direct activation of the alternative complement pathway by SARS-CoV-2 spike proteins is blocked by factor $D$ inhibition. Blood. 2020;136(18):2080-2089. doi:10.1182/blood.2020008248

37. Varga Z, Flammer AJ, Steiger $P$, et al. Endothelial cell infection and endotheliitis in COVID-19. Lancet. 2020;395(10234):1417-1418. doi:10.1016/50140-6736(20)30937-5

38. Goshua G, Pine AB, Meizlish ML, et al. Endotheliopathy in COVID19-associated coagulopathy: evidence from a single-centre, crosssectional study. Lancet Haematol. 2020;7(8):e575-e582. doi:10.1016/ S2352-3026(20)30216-7

39. Kuba K, Imai Y, Rao S, et al. A crucial role of angiotensin converting enzyme 2 (ACE2) in SARS coronavirus-induced lung injury. Nat Med 2005; 11(8): 875-879. doi: 10.1038/nm1267

40. Zhang H, Penninger JM, Li Y, Zhong N, Slutsky A. Angiotensinconverting enzyme 2 (ACE2) as a SARS-CoV-2 receptor: molecular mechanisms and potential therapeutic target. Intensive Care Med 2020; 46(4):586-590. doi: 10.1007/s00134-020-05985-9

41. Giannakopoulos B, Krilis SA. The pathogenesis of the antiphospholipid syndrome. N Engl J Med 2013; 368(11):1033-1044. doi: 10.1056/NEJMra1112830

42. Shatzel JJ, DeLoughery EP, Lorenz CU, et al. The contact activation system as a potential therapeutic target in patients with COVID-19. Res Pract Thromb Haemost 2020; 4(4): 500-505. doi: 10.1002/ rth2.12349

43. Moores LK, Tritschler T, Brosnahan S, et al. Prevention, Diagnosis, and Treatment of VTE in Patients With Coronavirus Disease 2019: CHEST Guideline and Expert Panel Report. Chest. 2020;158(3):11431163. doi:10.1016/j.chest.2020.05.559

44. Bikdeli B, Madhavan MV, Jimenez D, et al. COVID-19 and Thrombotic or Thromboembolic Disease: Implications for Prevention, Antithrombotic Therapy, and Follow-Up: JACC State-of-the-Art Review. J Am Coll Cardiol. 2020;75(23):2950-2973. doi:10.1016/j. jacc.2020.04.031

45. Barnes GD, Burnett A, Allen A, et al. Thromboembolism and anticoagulant therapy during the COVID-19 pandemic: interim clinical guidance from the anticoagulation forum. J Thromb Thrombolysis. 2020:50(1):72-81. doi:10.1007/s11239-020-02138-z

46. Nadkarni GN, Lala A, Bagiella E, et al. Anticoagulation, Bleeding, Mortality, and Pathology in Hospitalized Patients With COVID-19. J Am Coll Cardiol. 2020;76(16):1815-1826. doi:10.1016/j. jacc.2020.08.041

47. Hutt Centeno E, Militello M, Gomes MP. Anti-Xa assays: what is their role today in antithrombotic therapy? Cleve Clin J Med 2019; 86(6):417-425. doi: 10.3949/ccjm.86a.18029Abstract/FREE Full Text

48. Thachil J, Tang N, Gando S, et al. ISTH interim guidance on recognition and management of coagulopathy in COVID-19. J Thromb Haemost 2020; 18(5): 1023-1026. doi: 0.1111/jth.14810

49. Fox S, Dugar S. Point-of-care ultrasound and COVID-19 [published online ahead of print, 2020 May 14]. Cleve Clin J Med. 2020;10.3949/ccjm.87a.ccc019. doi:10.3949/ccjm.87a.ccc019

50. National Institutes of Health. Antithrombotic therapy in patients with COVID-19. Accessed July 10, 2020. https://www.covid19treatmentguidelines.nih.gov/antithrombotic-therapy/

51. Pedraza García J, Valle Alonso J, Ceballos García P, Rico Rodríguez F, Aguayo López MÁ, Muñoz-Villanueva MDC. Comparison of the accuracy of emergency department-performed point-of-careultrasound (POCUS) in the diagnosis of lower-extremity deep vein thrombosis. J Emerg Med 2018; 54(5):656-664. doi: 10.1016/j. jemermed.2017.12.020

52. Girardi G, Redecha P, Salmon JE. Heparin prevents antiphospholipid antibody-induced fetal loss by inhibiting complement activation. Nat Med 2004; 10(11):1222-1226. doi: 10.1038/nm1121

53. Poterucha TJ, Libby P, Goldhaber SZ. More than an anticoagulant: do heparins have direct anti-inflammatory effects? Thromb Haemost 2017; 117(3):437-444. doi: 10.1160/TH16-08-0620

54. Lang J, Yang N, Deng J, et al. Inhibition of SARS pseudovirus cell entry by lactoferrin binding to heparan sulfate proteoglycans. PLoS One 2011; 6(8):e23710. doi: 10.1371/journal.pone.0023710

55. Tang N, Bai H, Chen X, Gong J, Li D, Sun Z. Anticoagulant treatment is associated with decreased mortality in severe coronavirus disease 2019 patients with coagulopathy. J Thromb Haemost 2020; Mar 27. doi: 10.1111/jth.14817

56. White D, MacDonald S, Bull T, et al. Heparin resistance in COVID-19 patients in the intensive care unit [published correction appears in J Thromb Thrombolysis. 2020 Jun 22;:]. J Thromb Thrombolysis. 2020;50(2):287-291. doi:10.1007/s11239-020-02145-0

57. Paranjpe I, Fuster V, Lala A, et al. Association of treatment dose anticoagulation with in-hospital survival among hospitalized patients with COVID-19. J Am Coll Cardiol 2020 May 5; S07351097(20)35218-9. doi: 10.1016/j.jacc.2020.05.001FREE Full Text

58. Arachchillage DJ, Stacey A, Akor F, Scotz M, Laffan M. Thrombolysis restores perfusion in COVID-19 hypoxia. Br J Haematol. 2020;190(5):e270-e274. doi:10.1111/bjh.17050

59. Wang J, Hajizadeh N, Moore EE, et al. Tissue plasminogen activator (tPA) treatment for COVID-19 associated acute respiratory distress syndrome (ARDS): a case series. J Thromb Haemost 2020; Apr 8; 10.1111/jth.14828. doi: $10.1111 /$ jth. 14828

60. American Society of Hematology. COVID-19 resources. COVID-19 and coagulopathy: frequently asked questions. https://www.hematology.org/covid-19/covid-19-and-coagulopathy. Accessed June 18, 2020.

61. Palareti G, Cosmi B, Legnani C, et al; DULCIS (D-dimer and ULtrasonography in Combination Italian Study) Investigators. D-dimer to guide the duration of anticoagulation in patients with venous 
thromboembolism: a management study. Blood 2014; 124(2):

196-203. doi: 10.1182/blood-2014-01-548065Abstract/FREE Full Text

62. Cohen AT, Harrington RA, Goldhaber SZ, et al; APEX Investiga-

tors. Extended thromboprophylaxis with betrixaban in acutely ill medical patients. N Engl J Med 2016; 375(6): 534-544. doi: 10.1056/ NEJMoa1601747

Address: John R. Bartholomew, MD, Cardiovascular Medicine, ST2O, Cleveland Clinic, 9500 Euclid Avenue, Cleveland, OH 44195; barthoj@ ccf.org

Downloaded from www.ccjm.org on April 26, 2023. For personal use only. All other uses require permission. 